\title{
A bacterial interference strategy for prevention of UTI in persons practicing intermittent catheterization
}

\author{
A Prasad ${ }^{1}$, ME Cevallos ${ }^{2,3}$, S Riosa ${ }^{2,3}$, RO Darouiche ${ }^{2,3,4}$ and BW Trautner ${ }^{2,3,4}$ \\ ${ }^{1}$ Department of Internal Medicine, New York Medical College-Westchester Medical Center, Westchester, NY, USA; ${ }^{2}$ Division of \\ Infectious Diseases, Department of Medicine, Baylor College of Medicine, Houston, TX, USA; ${ }^{3}$ Department of Physical Medicine and \\ Rehabilitation, Center for Prosthesis Infection, Baylor College of Medicine, Houston, TX, USA and ${ }^{4}$ The Michael E DeBakey Veterans \\ Affairs Medical Center, Baylor College of Medicine, Houston, TX, USA
}

\begin{abstract}
Study design: Non-randomized pilot trial.
Objectives: To determine whether Escherichia coli 83972-coated urinary catheters in persons with spinal cord injury ( $\mathrm{SCl}$ ) practicing an intermittent catheterization program (ICP) could (1) achieve bladder colonization with this benign organism and (2) decrease the rate of symptomatic urinary tract infection (UTI).

Setting: Outpatient SCl clinic in a Veterans Affairs hospital (USA).

Methods: Participants had neurogenic bladders secondary to $\mathrm{SCl}$, were practicing ICP, had experienced at least one UTI and had documented bacteruria within the past year. All participants received a urinary catheter that had been pre-inoculated with $E$. coli 83972 . The catheter was left in place for 3 days and then removed. Participants were followed with urine cultures and telephone calls weekly for 28 days and then monthly until E. coli 83972 was lost from the urine. Outcome measures were $(1)$ the rate of successful bladder colonization, defined as the detection $\left(\geqslant 10^{2} \mathrm{cfu} \mathrm{ml}^{-1}\right)$ of $E$. coli 83972 in urine cultures for $>3$ days after catheter removal and (2) the rate of symptomatic UTI during colonization with E. coli 83972.

Results: Thirteen participants underwent 19 insertions of study catheters. Eight participants (62\%) became successfully colonized for $>3$ days after catheter removal. In these 8 participants, the rate of UTI during colonization was 0.77 per patient-year, in comparison with the rate of 2.27 UTI per patientyear before enrollment.

Conclusions: E. coli 83972-coated urinary catheters are a viable means of achieving bladder colonization with this potentially protective strain in persons practicing ICP.

Spinal Cord (2009) 47, 565-569; doi:10.1038/sc.2008.166; published online 13 January 2009
\end{abstract}

Keywords: urinary tract infection; spinal cord injury; Escherichia coli

\section{Introduction}

Each year, more than 30 million bladder catheters are used in the United States. ${ }^{1}$ Urinary catheters bypass normal host defenses to allow bacterial entry at a rate of approximately $3-10 \%$ per day, and they encourage bacterial persistence in the bladder. ${ }^{2}$ The majority $(98 \%)$ of individuals who are catheterized for 30 days or longer will have bacteriuria with one or more species of uropathogens. ${ }^{3}$ Individuals practicing an intermittent catheterization program (ICP) become colonized at a lower but still significant rate, with 51-61\% of urine samples from persons practicing ICP containing bacteria. $^{4,5}$ Although most episodes of catheter-associated bacteriuria are asymptomatic, asymptomatic bacteriuria

Correspondence: Dr BW Trautner, Department of Medicine, Baylor College of Medicine, One Baylor Plaza, BCM 286, N1319, Houston, TX 77030, USA. E-mail: trautner@bcm.edu

Received 18 September 2008; revised 23 October 2008; accepted 5 November 2008; published online 13 January 2009
(ABU) in many cases progresses to symptomatic urinary tract infection (UTI) and even bacteremia. ${ }^{6}$ Individuals with spinal cord injury (SCI) who rely on bladder catheters for urinary drainage are predisposed to recurrent UTI. ${ }^{7}$ Currently few, if any, measures are effective at preventing UTI in persons with neurogenic bladders secondary to SCI.

Bacterial interference, or using benign bacteria to prevent infection with virulent pathogens, ${ }^{8,9}$ may offer a solution to the significant problem of recurrent UTI in persons who require urinary catheters for bladder drainage. Urinary catheters which have been pre-inoculated with benign Escherichia coli 83972 may prevent UTI by interfering with either catheter colonization or bladder invasion by uropathogens. In vitro, we found that coating urinary catheters with E. coli 83972 impeded subsequent catheter colonization by a wide variety of uropathogens. ${ }^{10,11}$ Our earlier study in vivo with individuals with SCI using long-term, indwelling 
catheters also supports our hypothesis that E. coli-coated urinary catheters may help prevent catheter and bladder colonization by uropathogens and thus prevent UTI. As the preferred method of bladder management in persons with SCI is ICP, ${ }^{12,13}$ we studied the short-term use of $E$. coli 83972 coated urinary catheters as a means of achieving bladder colonization in persons with SCI practicing ICP. We also assessed the effect of bladder colonization with E. coli 83972 upon the incidence of symptomatic UTI.

\section{Methods}

\section{Participants}

This prospective clinical trial was approved by the institutional review board of Baylor College of Medicine and by the Research and Development Committee of the Michael E DeBakey Veterans Affairs Medical Center. Participants were recruited from the outpatient SCI clinic at this facility. Adults with SCI of $\geqslant 1$ year duration with neurogenic bladders managed by ICP were eligible for enrollment if they (1) had experienced at least one UTI in the past 10 years and (2) had documented bacteriuria within the past 1 year. Exclusion criteria included supravesicular urinary diversion, vesicoureteral reflux, obstructing urolithiasis, nephrostomy catheters, ongoing requirement for antibiotic therapy, poorly controlled diabetes mellitus, vascular or valvular prosthetic material, and immunosuppression. After providing informed consent, participants underwent either renal ultrasound or urodynamics to rule out obstructive urolithiasis and/or vesicoureteral reflux, and they submitted urine specimens for baseline cultures.

\section{Study design}

Each participant received an E. coli 83972-coated study catheter. Placement of a study catheter in the bladder was defined as an inoculation attempt. The catheter was left in place for 3 days and then removed and cultured by sonication. ${ }^{14}$ Three days was chosen as the dwell time, as this brief period of catheterization was convenient to participants (generally over the weekend) and also acceptable to health-care providers, as such a short dwell time was unlikely to change voiding dynamics. Urine cultures were performed on days $0,1,3,7,21,28$, and then monthly after catheter removal, until E. coli 83972 was lost from the urine. Participants were also called at these time intervals, to remind them to collect urine for the study and to question them about symptoms of UTI. Participants reporting symptoms that could be related to UTI were requested to come to the outpatient SCI clinic for evaluation by either the principal investigator or by their primary care physician. Participants who did not have E. coli 83972 in their urine or on their catheter on the day of study catheter removal could undergo another inoculation attempt, up to three attempts in total.

As we were interested in simplifying the inoculation protocol for the participants, we experimented with whether pre-inoculation antibiotics to clear the bladder of other flora were necessary to achieve successful bladder colonization with E. coli 83972. Pre-insertion antibiotics were not used for the first 10 inoculation attempts. As the rate of colonization was low in comparison with our earlier studies that had used pre-insertion antibiotics, the next nine inoculation attempts incorporated pre-insertion antibiotics. As per this protocol modification, participants who had detectable bacteriuria $\left(\geqslant 10^{2} \mathrm{cfu} \mathrm{ml}^{-1}\right)$ were treated for 7 days with antibiotics targeted at the organism(s) in the enrollment urine culture. Participants then had a 3- to 4-day washout period before study catheter insertion to allow antibiotics to clear from the urine.

\section{Catheter preparation and insertion}

To ensure patient safety, a pap deletion of wild-type E. coli 83972 was used to inoculate study catheters (Hu2117). ${ }^{15}$ Participants who required antibiotics to sterilize their bladder received study catheters prepared with a derivative strain of E. coli 83972 carrying short-term resistance to the appropriate antibiotic class (strains Hu2140, Hu2207, $\mathrm{Hu} 2209, \mathrm{Hu} 2293$ and Hu2194). In these derivative strains, the antibiotic resistance genes are carried on 'suicide' plasmids that are rapidly lost in the absence of selective pressure from antibiotics. Lubricious coated latex catheters (Bardex Lubricath, Covington, GA, USA) in trypticase soy broth (TSB; Becton Dickinson, Sparks, MD, USA) were each inoculated with a single colony of E. coli 83972 and then incubated at $37^{\circ} \mathrm{C}$ static for $48 \mathrm{~h}$. The composition of the lubricious coating is proprietary, but this hydrophilic surface became slippery during the catheter preparation process, thus facilitating insertion. At 24 and $48 \mathrm{~h}$, the broth was tested for contamination by dilution plating. After $48 \mathrm{~h}$ of incubation, the catheter was inserted into the participant's bladder under otherwise sterile conditions. Participants generally placed their own catheters.

\section{Outcomes}

The outcomes of interest were (1) the rate of successful bladder colonization and (2) the rate of symptomatic UTI during colonization with E. coli 83972. Successful bladder colonization was defined as the detection $\left(\geqslant 10^{2} \mathrm{cfu} \mathrm{ml}^{-1}\right)$ of E. coli 83972 in urine cultures for $>3$ days after catheter removal. Pre-insertion antibiotics were considered to have been effective if the urine culture collected immediately before catheter insertion grew $\leqslant 10^{2} \mathrm{cfu} \mathrm{ml}^{-1}$ organisms.

For each participant, we documented the incidence of symptomatic UTI during colonization with E. coli 83972. Symptomatic UTI was defined as significant bacteriuria $\left(\geqslant 10^{5} \mathrm{cfu} \mathrm{ml}^{-1}\right)$ and pyuria ( $>10 \mathrm{WBC}$ per high power field) plus $\geqslant 1$ of the following signs and symptoms for which no other etiology could be identified: fever (oral temperature $>100^{\circ} \mathrm{F}$ ), suprapubic or flank discomfort, bladder spasm, change in voiding habits, increased spasticity or worsening dysreflexia. ${ }^{16,17}$ The determination of symptomatic UTI was made by the research team together with the primary care provider based upon participants' self-reported symptoms. The rate of UTI per participant per year during colonization was calculated by extrapolating the total number of participant days of colonization to 1 year. Only participants 
who became colonized for $>3$ days were included in this calculation. To have a baseline for comparison, we also recorded the number of UTI occurring during the year before study enrollment and during the year after bladder colonization (including the time period of colonization). These UTIs were determined by a retrospective chart review by the principal investigator. The definition of UTI used for retrospective ascertainment of UTI was the presence of symptoms attributable to the urinary tract, documented bacteriuria and the administration of antibiotics to treat UTI. As two participants had only 8 months of follow-up data, the overall UTI incidence was calculated per available months of follow-up and converted to the patient-year measurement.

\section{Results}

All 13 participants were men, had neurogenic bladder secondary to SCI and were using ICP for bladder management. Their average age was 56 years (range 37-73 years), and the average time since injury was 15 years (range 1.3-35 years). Seven were white $(54 \%)$, whereas six were black (46\%). Nineteen inoculation attempts were made in these 13 participants, resulting in eight bladder colonizations lasting for $>3$ days. Thus, $62 \%$ of participants became colonized. The overall success rate of a single inoculation attempt was $42 \%$.

Before the use of pre-insertion antibiotics, 10 inoculation attempts resulted in three bladder colonizations. Subsequently, eight participants underwent nine inoculation attempts under the pre-insertion antibiotic protocol, resulting in five successful colonizations. It is worth noting that two participants (nos. 6 and 7) underwent two inoculation attempts each, one without and one with pre-insertion antibiotics. The success rate of inoculation after pre-insertion antibiotics was 55\%, in comparison with the success rate without pre-insertion antibiotics of 33\% $(P=0.26$, Fisher's
Exact Test). Pre-insertion antibiotics were ineffective at sterilizing the urine despite the fact that we chose antibiotics based on the antimicrobial susceptibilities of the organisms in an enrollment urine culture. Specifically, of the seven participants who received antibiotics under the pre-insertion antibiotics protocol (one participant had sterile urine at enrollment), six had bacteriuria at the time of study catheter insertion, usually with one or more new species (Table 1).

The pre-study rate of UTI for all participants was 2.27 UTI per subject-year and was 2.29 UTI per subject-year for participants who subsequently became successfully colonized (excluding the participants for whom UTI per year was unknown). For the eight participants who became successfully colonized, the rate of symptomatic UTI requiring treatment during colonization with E. coli 83972 was 0.77 per patient-year. During the year after the insertion of study catheters (excluding the time period of colonization with E. coli 83972), the rate of UTI for all participants was 1.98 UTI per subject-year. Only one participant (no. 2) experienced a symptomatic UTI during potential colonization with $E$. coli 83972. On day 97 after study catheter removal, his urine culture contained Proteus and E. coli 83972. He inserted an indwelling foley catheter the next day for convenience in bladder management. Two days later he developed fever, chills and hematuria. Urine collected on this day before initiation of antibiotics grew Proteus and a pathogenic E. coli strain. No E. coli 83972 was present in this urine culture, but we included this UTI as an event occurring during colonization in the interest of avoiding bias. It is likely that his UTI was related to the change in his bladder management, as a urinary catheter provides an excellent substrate for Proteus growth. ${ }^{18}$ We have also observed earlier that Proteus tends to eliminate E. coli 83972 in the catheterized human bladder. ${ }^{19}$

The case of participant no. 12 is illustrative of a potential protective effect of bladder colonization by E. coli 83972 .

Table 1 Colonization success and UTI outcomes

\begin{tabular}{|c|c|c|c|c|c|c|c|c|c|}
\hline Subject & $\begin{array}{c}\text { Pre-study } \\
\text { UTI } \\
\text { per year }\end{array}$ & $\begin{array}{l}\text { Pre-insertion } \\
\text { antibiotics }\end{array}$ & $\begin{array}{l}\text { Colonization } \\
\text { attempts }\end{array}$ & $\begin{array}{l}\text { Organisms present } \\
\text { at insertion }\end{array}$ & $\begin{array}{l}\text { Days } \\
\text { colonized }\end{array}$ & $\begin{array}{l}\text { Successful } \\
\text { colonization? }\end{array}$ & $\begin{array}{l}\text { No. of } \\
\text { UTI while } \\
\text { colonized }\end{array}$ & $\begin{array}{l}\text { Reason for } \\
\text { loss of } 83972\end{array}$ & $\begin{array}{l}\text { Post-study } \\
\text { UTI per year }\end{array}$ \\
\hline 1 & 1 & No & 1 & Enterococcus & 197 & Yes & 0 & Spontaneous & 1 \\
\hline 2 & 2 & No & 3 & Proteus & 97 & Yes & $1^{a}$ & Antibiotics & 3 \\
\hline 3 & Unknown ${ }^{\mathrm{b}}$ & No & 1 & Serratia Enterococcus & 0 & No & 0 & NA & Unknown ${ }^{b}$ \\
\hline 4 & 0 & No & 2 & Pseudomonas Klebsiella & 11 & Yes & 0 & Spontaneous & 2 \\
\hline 5 & 0 & No & 1 & Klebsiella & 0 & No & 0 & NA & 0 \\
\hline \multirow[t]{2}{*}{6} & 1 & No & 1 & Escherichia coli & 0 & No & NA & NA & 2 \\
\hline & & Yes & 1 & E. coli & 0 & No & & & \\
\hline \multirow[t]{2}{*}{7} & Unknown ${ }^{\mathrm{b}}$ & No & 1 & E. coli & 0 & No & NA & NA & Unknown ${ }^{b}$ \\
\hline & & Yes & 1 & Klebsiella and Providencia & 28 & Yes & 0 & Spontaneous & \\
\hline 8 & 2 & Yes & 1 & None & 21 & Yes & 0 & Spontaneous & 2 \\
\hline 9 & 1 & $\begin{array}{l}\text { None } \\
\text { indicated }\end{array}$ & 1 & None & 10 & Yes & 0 & Antibiotics & 2 \\
\hline 10 & 5 & Yes & 1 & Pseudomonas & 71 & Yes & 0 & Spontaneous & 0 \\
\hline 11 & 4 & Yes & 2 & Citrobacter (both times) & 0 & No & NA & NA & 0 \\
\hline 12 & 5 & Yes & 1 & Pseudomonas and Enterococcus & 39 & Yes & 0 & Antibiotics & $4^{c}$ \\
\hline 13 & 4 & Yes & 1 & Klebsiella & 0 & No & NA & NA & $2^{c}$ \\
\hline
\end{tabular}

Abbreviations: NA, not applicable; UTI, urinary tract infection.

${ }^{a}$ This subject did not have E. coli 83972 in his urine on the day of UTI diagnosis, but had been colonized with E. coli 839722 days before.

bUnknown means that the subject received the majority of his care at an outside facility, so the number of UTIs could not be documented accurately.

'These subjects had 8 months of follow-up after inoculation. 
During colonization with E. coli 83972, he did not experience urinary symptoms despite the presence of Pseudomonas in his urine. This $\mathrm{ABU}$ was inappropriately treated with antibiotics on day 39 after catheter removal. These antibiotics eradicated E. coli 83972 but not the Pseudomonas. He subsequently developed bladder spasms and required targeted therapy to eradicate the Pseudomonas.

Our bladder colonization protocol was well tolerated. Three participants experienced adverse effects: groin pain during colonization that resolved spontaneously, dysreflexia during inflation of the catheter balloon and symptomatic UTI in a participant who failed to become colonized.

\section{Discussion}

E. coli 83972-coated urinary catheters are a viable means to achieve bladder colonization with this benign organism. This study is too small to determine which factors predicted successful colonization, but pre-inoculation antibiotics tended to increase the colonization success rate. Although these antibiotics did not clear the bladder of other flora, we suspect that they suppressed the quantity of other organisms and helped E. coli 83972 become established. However, overall, only 8 of 19 inoculation attempts resulted in successful colonization. We would expect a higher success rate of colonization if participants underwent successive placement of study catheters, or possibly if we increased the dwell time of the catheters as in our study in the long-term catheterized SCI participants. ${ }^{19}$

Our study confirms the futility of treating ABU in persons with SCI. When we treated participants' baseline ABU to facilitate bladder colonization, only one of seven treated participants had sterile urine following a course of antibiotics to which the organisms cultured from their urine were susceptible. Thus, we agree with guidelines that advise non-treatment of ABU in the SCI population. ${ }^{20}$

Our data suggest that participants have protection from symptomatic UTI during colonization with E. coli 83972, although the use of a retrospective, historical control is a potential source of bias. Over diagnosis of UTI in the preceding year may give an appearance of efficacy when in fact none is present. We can definitively report that the number of treated episodes of UTI decreased when participants were colonized with E. coli 83972 in comparison with the year before the study and with the year after inoculation (excluding the time of colonization with E. coli 83972). However, this difference may be attributed to differences in case ascertainment, as we actively followed participants for the occurrence of UTI during colonization, while determination of UTI during other time periods was based on chart review. As the pre-study rate of UTI (2.27 UTI per year) is similar to the rate of UTI after loss of colonization with E. coli 83972 (1.98 UTI per year), we believe that we successfully applied the same case definitions throughout.

Another potential weakness in our study is the lack of placebo control. Bladder inoculation with an E. coli 83792coated urinary catheter was likely to make a strong impression on the research participants and could have influenced their reporting of symptoms of UTI. The research team also knew of the participants' colonization status. This work was designed as a pilot study and will help us plan the appropriate randomized, placebo-controlled trial. All of our participants were men, as $99 \%$ of the SCI population at our facility are men, so whether our results are applicable to women with SCI is undetermined.

Overall, short-term use of E. coli 83972-coated urinary catheters was an effective, safe and well-tolerated means to achieve bladder colonization with E. coli 83972. Participants may have a decreased risk of symptomatic UTI during colonization with this organism. The possible protective effect against UTI encourages us to perform further trials with E. coli 83972-coated urinary catheters in the SCI population, including persons practicing ICP.

\section{Acknowledgements}

This study was supported by Department of Veterans Affairs Rehabilitation Research and Development Service grants B4623R, B4087R and B4387P as well as by NIH grants HD42014 and DK77313.

\section{References}

1 Darouiche R. Device-associated infections: a macroproblem that starts with microadherence. Clin Infect Dis 2001; 33: 1567-1572.

2 Warren J. Catheter-associated urinary tract infections. Infect Dis Clin North Am 1997; 11: 609-622.

3 Warren J, Tenney J, Hoopes J, Muncle H, Anthony W. A prospective microbiologic study of bacteriuria in patients with chronic indwelling urethral catheters. I Infect Dis 1982; 146: 719-723.

4 Bakke A, Digranes A. Bacteriuria in patients treated with clean intermittent catheterization. Scand J Infect Dis 1991; 23: 577-582.

5 Bakke A, Digranes A, Hoisaeter P. Physical predictors of infection in patients treated with clean intermittent catheterization: a prospective 7-year study. Br J Urol 1997; 79: 85-90.

6 Saint S. Clinical and economic consequences of nosocomial catheter-related bacteriuria. Am J Infect Control 2000; 28: 68-75.

7 Darouiche R. Infections in patients with spinal cord injury. In: Mandell G, Bennett J, Dolin R (eds). Principles and Practice of Infectious Disease, 6th edn. Churchill Livingstone: Philadelphia, 2004, pp 3512-3517.

8 Sprunt K, Leidy G. The use of bacterial interference to prevent infection. Can J Microbiol 1988; 34: 332-338.

9 Bibel D. Bacterial interference, bacteriotherapy, and bacterioprophylaxis. In: Aly $\mathrm{R}$, Shinefield $\mathrm{H}$ (eds). Bacterial Interference. CRC Press: Boca Raton, 1982, pp 1-12.

10 Trautner B, Darouiche R, Hull R, Hull S, Thornby J. Preinoculation of urinary catheters with Escherichia coli 83972 inhibits catheter colonization by Enterococcus faecalis. I Urol 2002; 167: 375-379.

11 Trautner B, Hull R, Darouiche R. Escherichia coli 83972 inhibits catheter adherence by a broad spectrum of uropathogens. Urology 2003; 61: 1059-1062.

12 Bennett C, Young M, Adkins R, Diaz F. Comparison of bladder management complication outcomes in female spinal cord injury patients. J Urol 1995; 153: 1458-1460.

13 Weld K, Dmochowski R. Effect of bladder management on urological complications in spinal cord injured patients. J Urol 2000; 163: 768-772.

14 Sherertz R, Raad I, Belani A, Koo L, Rand K, Pickett D et al. Three-year experience with sonicated vascular catheter cultures in a clinical microbiology laboratory. J Clin Microbiol 1990; 28: 76-82. 
15 Hull R, Donovan W, DelTerzo M, Stewart C, Rogers M, Darouiche R. Role of type 1 fimbria- and P fimbriae-specific adherence in colonization of the neurogenic human bladder by Escherichia coli. Infect Immun 2002; 70: 6481-6484.

16 Darouiche R, Thornby J, Cerra-Stewart C, Donovan W, Hull R. Bacterial interference for preventing urinary tract infection: a prospective, randomized, placebo-controlled, double-blind pilot trial. Clin Infect Dis 2005; 41: 1531-1540.

17 Darouiche R, Donovan W, Del Terzo M, Thornby J, Rudy D, Hull R. Pilot trial of bacterial interference for preventing urinary tract infection. Urology 2001; 58: 339-344.
18 Stickler D, Ganderton L, King J, Nettleton J, Winters C. Proteus mirabilis biofilms and the encrustation of urethral catheters. Urol Res 1993; 21: 407-411.

19 Trautner BW, Hull RA, Thornby JI, Darouiche RO. Coating urinary catheters with an avirulent strain of Escherichia coli as a means to establish asymptomatic colonization. Infect Control Hosp Epidemiol 2007; 28: 92-94.

20 Nicolle L, Bradley S, Colgan R, Rice JC, Schaeffer A, Hooten TM. Infectious Diseases Society of America guidelines for the diagnosis and treatment of asymptomatic bacteriuria in adults. Clin Infect Dis 2005; 40: 643-654. 